\title{
Introduction to the special issue on intelligent metaheuristics for logistic planning
}

\author{
Belén Melián-Batista • José A. Moreno-Pérez
}

Received: 25 February 2013 / Revised: 1 March 2013 / Accepted: 5 March 2013 /

Published online: 16 March 2013

(C) Springer Science+Business Media New York 2013

Intelligent approaches for solving the real-world problems that arise in logistic planning generally include the use of Metaheuristics, which are among the most efficient and robust methods to find high-quality solutions to a large number of optimization problems. The intelligent qualificative is assigned to metaheuristics because of the fact that they might incorporate machine learning tools, use short, medium and long term memory, include self-adaptation of their parameters, incorporate expert knowledge to improve their performance, etc. These and other features of metaheuristics make them increasingly important for designing decision support systems in the planning of logistics activity at the strategic, tactical and operational levels. Furthermore, the realworld problems which are tackled by the managers of logistics activities have special characteristics that recommend the use of metaheuristics as well as the research on the best way to implement them.

Since the notion of metaheuristics was introduced by Fred Glover in 1986, numerous applications have appeared in the literature. This conceptual framework includes a number of methodologies for tacking difficult optimization problems that originated before and after the term metaheuristic was coined, for instance, genetic algorithms and simulated annealing. Currently, the field of metaheuristics is one of the research areas of the artificial intelligence with the greatest impact in terms of applications to real situations of business interest.

The development and application of metaheuristics, unlike heuristics specifically designed for a problem, seek efficacy, efficiency and robustness across problem classes. These characteristics, under the guidance of expert systems, have enabled the

B. Melián-Batista $(\varangle)$ J J. A. Moreno-Pérez

ETS de Ingeniería Informática, University of La Laguna, Santa Cruz de Tenerife, Spain

e-mail:mbmelian@ull.es

J. A. Moreno-Pérez

e-mail: jamoreno@ull.es 
embedding of these methods in decision support systems of companies and institutions with responsibilities in logistics activity.

Logistics planning involves making fundamental decisions at three main levels: strategic, tactical and operational. Strategic decisions have planning horizons of months or years. Some of the most common strategic decisions include warehouse design, warehouse location or issues related to the political aspects of distribution. Tactical decisions are made every few months or weeks, or have an effect within a similar time period. Among the most common tactical issues are the activity zoning and those that set some elements of the distribution networks. Finally, operational decisions are made daily or every few days and the effect is immediate. Among the most important operational decisions are vehicle routing and load management. These three decision levels require efficiency and effectiveness of the associated optimization procedures and metaheuristics provide the appropriate support for all of them.

Furthermore, metaheuristic methodologies are capable of dealing effectively with the uncertainty associated with many real-world problems. Interesting applications of metaheuristics to relevant problems in logistics frequently appear in both academic and practitioner-oriented publications. Therefore, businesses show an increasing interest in incorporating these intelligent tools into their applications that support logistics activities.

This special issue is devoted to report on the latest advances in intelligent metaheuristics applied to real-world logistics problems. The contents of this issue illustrate breadth and depth of research in both metaheuristics and the various areas of logistics. The main contributions focus on applications as well as on the development of new features of metaheuristic solution approaches. The initial idea for editing this issue came at the Workshop on Intelligent Metaheuristics for Logistic Planning that took place during the Conference of the Spanish Association for Artificial Intelligence (CAEPIA 2009), which was held in Seville on the 13th of November of 2009. Some of the thirteen articles in this issue were presented in that workshop.

Julio Mar-Ortiz, José Luis González-Velarde and Belarmino Adenso-Díaz propose an integer programming model and describe a GRASP-based algorithm to solve a vehicle routing and scheduling problem for the collection of Waste of Electric and Electronic Equipment (WEEE). The most important contribution of this paper to the logistics literature is that the problem is characterized by four variants of the vehicle routing problem that have been independently studied in the literature, but have not been considered all together. The experimental analysis on a large set of randomlygenerated instances shows the good performance of the proposed algorithm. Moreover, computational results using real data show that the method outperforms real existing approaches to reverse logistics.

Vera Hemmelmayr, Karl F. Doerner, Richard F. Hartl and Stefan Rath study a realworld waste collection problem in which glass, metal, plastics or paper is brought to waste collection points in region. The collection of this waste is therefore a node routing problem. The waste is delivered to special sites, called intermediate facilities (IF), that are typically not identical to the vehicle depot. Since most waste collection points need not be visited every day, a planning period of several days has to be considered. Thus, three interrelated planning problems must be considered: the periodic vehicle routing problem with intermediate facilities (PVRP-IF), the PVRP-IF modified by permitting 
the return of partly loaded vehicles to the depots and by considering capacity limits at the depots, and the multi-depot vehicle routing problem with inter-depot routes (MDVRPI), for which just a single day is considered and the depots can act as an intermediate facility only at the end of a tour. For the first case, a mathematical problem formulation is proposed, a set of benchmark instances is developed, and a hybrid solution method based on variable neighborhood search and dynamic programming is presented. For the second problem, an average improvement of $25 \%$ in the routing cost is obtained compared to the current solution. Finally, for the MDVRPI there are available several instances and benchmark solutions. It is shown that the algorithm outperforms all previously published metaheuristics for this problem class and finds the best solutions for all available benchmark instances.

Irma García, Joaquín Pacheco and Ada Alvarez propose a real-world application posed to the authors by a bakery company in Northern Spain. The problem consists of designing daily routes for a week in order to minimize the total distance. Flexibility on the delivery dates causes a stock and the problem is studied under a bi-objective perspective. The goal is to minimize both the total distance and the stock. A bi-objective mixed-integer linear model for the problem is formulated and two solution methods are presented. The first one is based on a series of linked variable neighborhood searches and the second one is based on NSGA-II. Numerical results show the obtained estimated Pareto fronts in both cases.

Angélica Salazar-Aguilar, Roger Z. Ríos-Mercado and J. L. González-Velarde deal with a real-world logistic problem. Particularly, a bi-objective commercial territory design problem motivated by an application from the bottled beverage distribution industry is addressed. Territory compactness and balancing with respect to the number of customers are considered as optimization criteria. Contrary to previous research, in which exact methods for small to medium-scale instances have been proposed, this work develops a GRASP framework for tackling considerably large instances. The main contributions of this paper rely on a real-world application relevant to the industry logistics and on the development of advanced GRASP-based procedures (BGRASP and TGRASP). These strategies are empirically evaluated and compared to NSGA-II, one of the most successful evolutionary methods known in the literature. Computational results show the superiority of the proposed strategies. In addition, one of the proposed GRASP strategies is successfully applied to a case study from industry.

Puca Huachi Vaz Penna, Anand Subramanian and Luiz Satoru Ochi tackle the Heterogeneous Fleet Vehicle Routing Problem (HFVRP), in which customers are served by a heterogeneous fleet of vehicles with different capacities and costs. The goal is to design a set of routes in such a way that the sum of the costs is minimized. HFVRP is NP-hard since it is a generalization of the classical VRP. This work proposes an algorithm based on the Iterated Local Search (ILS) metaheuristic, which uses a Variable Neighborhood Descent procedure with a random neighborhood ordering (RVND) in the local search phase. The method was tested on well-known benchmark instances providing results that are quite competitive when compared to other algorithms from the literature.

Jair J. De la Cruz, Carlos D. Paternina-Arboleda, Victor Cantillo and Jairo R. Montoya-Torres deal with the Heterogeneous Vehicle Routing Problem with Time 
Windows and Multiple Products (HVRPTWMP). Since the problem is NP-hard, most of the literature research has been focused on the design of heuristics and metaheuristics. The solution approach proposed in this work is a sequential Ant Colony System (ACS)—Tabu Search algorithm, which introduces a two pheromone trail strategy to accelerate the agents learning process. Its convergence to good solutions is given in terms of fleet size and travel time while completing tours and service to all customers. The proposed procedure uses different forms of memory from tabu search to further improve solution quality. Experiments are carried out over instances from the related literature and show the effectiveness of the procedure.

Carlos Gracia, Carlos Andrés and Luis Gracia present a hybrid approach based on the use of genetic algorithms to efficiently solve the real-world problem of cutting structural beams arising at a metalwork company. The problem belongs to the class of one-dimensional multiple stock sizes cutting stock problem. The proposed approach handles overproduction and underproduction of beams and embodies the reusability of remnants in the optimization process. Along with genetic algorithms, the approach incorporates other novel refinement algorithms that are based on different search and clustering strategies. The approach is experimentally tested on a set of instances based on the real ones. Computational results show that the proposed approach substantially improves the performance of previous heuristics.

Sara Ceschia and Andrea Schaerf consider a complex variant of the Container Loading Problem, also arising from a real-world industrial application. It includes several features, which are important in many practical situations. They propose a solution technique based on local search metaheuristics, which work on the space of sequences of boxes to be loaded, while the actual load is obtained by invoking, at each iteration, a specialized procedure called loader. The loader inserts the boxes in the container using a deterministic heuristic, which produces a load that is feasible according to the constraints. The algorithm is tested on real-world instances provided by the industrial partner, showing a clear improvement on the previous heuristic solution. It is also compared with the literature related to basic container loading problems. The method produces competitive results when compared to those that have appeared in the literature.

Marco Caserta and Stefan Vo present a framework to tackle mixed integer programming problems based upon a "constrained" black box approach. Given a MIP formulation, a black-box solver, and a set of incumbent solutions, they iteratively build corridors around such solutions by adding exogenous constraints to the original MIP formulation. Such corridors, or neighborhoods, are then explored with a standard MIP solver. An iterative approach is thus used to explore subportions of the solution space. The proposed framework has been tested on a challenging variation of the lot sizing problem, the multi-level lot sizing problem with setups and carryovers. The algorithm was effective, in terms of solution quality as well as computational time, when compared with a commercial MIP solver and the best algorithm from the literature. The main contribution of this paper is methodological.

Rong Qu, Ying Xu, Juan P. Castro, Dario Landa-Silva apply a Particle Swarm Optimization (PSO) algorithm to both the Steiner tree problem and the delay constrained multicast routing problem. Steiner tree problems, as the underlining models of many applications, have received significant research attention within the 
metaheuristics community. The literature on the application of meta-heuristics to multicast routing problems is less extensive but includes several promising approaches. Many interesting research issues still remain to be investigated; for instance, the inclusion of different constraints, such as delay bounds, when finding multicast trees with minimum cost. This work develops a novel PSO algorithm and also proposes two novel local search heuristics within the PSO framework. A path replacement operator is used in particle moves to improve the positions of the particle with regard to the structure of the tree. The performance of the PSO algorithm and the effect of the integrated local search heuristics are tested by an extensive set of experiments on multicast routing benchmark problems and Steiner tree problems from the OR library. The experimental results show the superiority of the proposed PSO algorithm over a number of other state-of-the-art approaches.

Julián Aráoz, Elena Fernández and Carles Franquesa address the Clustered Prize Collecting Arc Routing Problem, which is an arc routing problem where each demand edge is associated with a profit that is collected once if the edge is serviced, independently of the number of times it is traversed. It is further required that if a demand edge is serviced, then all the demand edges of its component are also serviced. The main contribution of this work is the development of GRASP and Path Relinking heuristics for the problem at hand, in which the solutions generated by two alternative versions of GRASP are used as elite solutions for the path relinking. The results of extensive computational experiments are presented and analyzed.

Deniz Aksen, Necati Aras and Nuray Piyade tackle a bilevel p-median problem for the planning and protection of critical facilities, which involves a static Stackelberg game between a system planner and a potential attacker. The system planner first determines where to open critical service facilities and then decides which of them to protect with a limited protection budget. Following this twofold action, the attacker decides which facilities to interdict simultaneously, where the maximum number of interdictions is fixed. The authors provide a bi-level integer program (BIP) for this problem, in which the defender takes on the leader role and the attacker acts as the follower. Then, three different methods are proposed: an optimal exhaustive search algorithm with exponential time complexity; a two-phase tabu search heuristic developed to overcome the computational burden of the first method; and a sequential solution method in which the defender location and protection decisions are separated. The results show that protection budget plays a significant role in maintaining the service accessibility of critical facilities in the worst-case interdiction scenario.

Bosco García-Archilla, Antonio J. Lozano, Juan A. Mesa and Federico Perea analyze the solvability of a railway network design problem and its robust version. These problems are modeled as integer linear programming problems, whose solutions provide topological railway networks maximizing the trip coverage in the presence of a competing mode, both assuming that the network works fine and that links can fail, respectively. Since these problems are computationally intractable for realistic sizes, GRASP heuristics are proposed for finding good feasible solutions. The results obtained in the computational experience indicate that the proposed GRASP algorithms are suitable for railway network design problems. 